\title{
CONVERGENCE MÉDIATIQUE ET VISIBILITÉ NUMÉRIQUE VATICANE : LE DÉVELOPPEMENT DE VATICAN NEWS
}

\begin{abstract}
Angela AnZelmo ${ }^{1}$
Depuis la fin des années 1990, l'Église catholique s'est emparée des dispositifs numériques pour transmettre un message politico-religieux d'une modernité nécessaire pour communiquer et demeurer visible à l'heure des hypermédias. Une avancée communicationnelle se dessine depuis 2018, notamment par la convergence médiatique de ce qui constituait précédemment un appareil communicationnel numérique fractionné, voire divisé, dans lequel les entités médiatiques du Dicastère pour la communication se juxtaposaient. Or à la lumière de la pensée d'Henri Jenkins, quelle(s) conséquence(s) engendre la convergence médiatique en termes de décloisonnement des publics et, a fortiori, de visibilité institutionnelle ? Quelles valeurs médiatiques le web 2.0 introduit-il pour les processus de traduction des discours institutionnels d'une communauté à l'autre ? Cette contribution se propose de répondre à ces questions en étudiant les moyens investis pour permettre à l'Église catholique de gagner en efficacité communicationnelle depuis les trois dernières années.
\end{abstract}

\section{Introduction}

Cet article se fonde sur une épistémologie sémiotique pour étudier les pratiques numériques vaticanes dans le contexte de la convergence

1 Angela Anzelmo est docteure en Sémiotique et Communication, associée au Centre de Recherches Sémiotiques (Limoges) et membre du Laboratorio Romano di Semiotica (Rome, Italie).

Recherches en communication, $\mathrm{n}^{\circ} 53$ - Article publié le 15/09/2021 
médiatique du Dicastère pour la communication. Objet d'intérêt pour le champ des Digital Religion Studies, qui se fondent sur les études d'internet, des médias, de la religion et de la culture (Campbell, 2017), il s'agit d'étudier le processus de convergence des publics engendré par celle des médias du Vatican, en privilégiant l'approche médiologique (notamment les travaux de Régis Debray ${ }^{2}$ et de Daniel Bougnoux ${ }^{3}$ ) pour étudier les valeurs de médiation ${ }^{4}$.

L'objectif global de la réforme des médias de l'Église catholique montre un premier résultat - le lancement du nouveau portail Vatican News - reflétant un changement de perspective, le passage d'une conception médiatique fractionnée à une conception multimédia. Une telle réforme s'accompagne d'un réaménagement propre à maintenir le leadership de l'institution religieuse dans le contexte communicationnel actuel.

En partant du principe que le média ne se réduit pas aux discours et qu'il est indispensable de prendre en compte le potentiel de communication qu'il offre " en fonction de ses caractéristiques techniques » (Papi, 2018, p. 105), nous entendons étudier l'opérationnalité des médiations en fonction des dispositifs (sites web, applications mobiles et réseaux sociaux) via l'expérience-usager qu'ils offrent (boutons, liens hypertextes, technomots ${ }^{5}$ ). Il s'agit plus exactement d'examiner la structure sémiotique des pratiques numériques et leurs « manifestations observables, énonciations, elles-mêmes analysables en contenus de signification » (Fontanille, 2006, p. 8). La seule focalisation sur les technologies d'information, et donc sur l'objet, ne rend visible ni les circulations ni les productions (Souchier et al., 2003, p. 39), et même si tous les médias sont à un certain niveau " signifiants " notamment lors des processus de remédiation (Bolter \& Grusin, 1999, p. 19), plus spécifiquement, c'est le champ des pratiques qui permet d'y observer le

2 Debray, R (2000). Introduction à la médiologie. Paris : PUF.

3 Bougnoux, D. (1995). La Communication contre l'information. Coll. Questions de Société. Paris : Hachette.

4 La valeur de médiation selon Eleni Mitropoulou (2015, p. 595), constitue l'entrée nécessaire pour une comparaison entre dispositifs.

5 Selon Marie-Anne Paveau (2017, p. 117) les « technomots sont nativement et directement cliquables soit à partir des programmes d'écriture des plateformes (nom des comptes de réseaux sociaux, pseudo Twitter, résultat de recherche, mot-consigne), soit à partir d'un geste d'écriture tel que le hashtag, le tag. Il dirige l'internaute vers des comptes, des fils, des redocuments $»$. 
développement des médiations (Jeanneret, 2014, p. 60). Afin de saisir le caractère intentionnel de ces pratiques, nous convoquerons à l'analyse les commentaires de Dario Edoardo Viganò, assesseur au Dicastère pour la communication, recueillis lors d'un entretien semi-directif.

L'édition de la plateforme Vatican News sur laquelle convergent les productions de deux médias institutionnels que sont Radio Vatican et Vatican Media (anciennement CTV), entraîne parallèlement des modifications importantes dans la manière même de communiquer, en valorisant l'isotopie de l'inclusion ${ }^{6}$ (Peverini, 2017, p. 117), ce qu'Henri Jenkins nomme " convergence des publics ». Nous verrons dans quelles mesures la technique permet désormais à l'institution de s'adresser simultanément à des individus de toutes communautés linguistiques, qu'ils soient fidèles ou profanes, ambitionnant ainsi une communication universelle.

Notre corpus se compose des anciens sites web (Radio Vatican, Osservatore Romano et $C T V$ ), antérieurs à la réforme des médias et du site Vatican News ${ }^{7}$ créé pour la convergence médiatique. Les comptes Twitter, Instagram et Facebook de Vatican News (y compris le compte newsva " ancêtre » de Vatican News sur Instagram) seront convoqués ainsi que l'application mobile Vatican Audio ${ }^{8}$. Le critère de choix ayant motivé un tel corpus concerne essentiellement le passage d'une structure communicationnelle fractionnée, voire « éparpillée » à une organisation médiatique convergée.

Nous débuterons en exposant la réforme des médias ainsi qu'un état des lieux de ce que constituait la communication numérique avant (de 2016 à 2018) et après (de 2018 à 2020) la convergence médiatique. Nous verrons ensuite en quoi la convergence des publics participe à propulser le discours politico-religieux au-delà des frontières linguistiques et culturelles du public dans la mesure où les nouvelles pratiques numériques de l'Église catholique permettent, dans la conception vati-

6 Peverini traitait en 2017 essentiellement du nouveau portail. Nous souhaitons élargir l'analyse à d'autres dispositifs pour montrer que l'intention est la même, globale, et ne regarde pas seulement le site web Vatican News.

7 En ligne depuis janvier 2018, il correspond au résultat de la fusion des médias du Vatican (www.vaticannews.va).

8 Désormais disparue, elle était en service jusqu'à mai 2019 avant d'être confondue à celle de Radio Vatican.

9 Dans le cadre notre thèse de doctorat, nous nous sommes intéressée à partir de 2016 à la communication vaticane. 
cane, une perméabilité discursive pour parler potentiellement à tous les hommes.

\section{Réforme et convergence des médias du Vatican}

Pour répondre au contexte communicationnel actuel, caractérisé par la présence et le développement des médias numériques, par la convergence et l'interactivité, le pape François avait institutionnalisé, le 27 juin 2015, un nouveau dicastère, le Dicastère pour la communication (anciennement appelé « Secrétariat pour la communication »). Le texte du Motu proprio ${ }^{10}$ stipulait ceci :

Le contexte actuel de la communication, caractérisé par la présence et le développement des médias numériques [...] exige de revoir le système d'information du Saint-Siège et conduit à une réorganisation qui, tout en valorisant ce qui a été développé au cours de l'histoire au sein du secteur de la communication du Siège apostolique, procède de façon résolue vers une intégration et une gestion unitaire (François, 2015).

L'objectif est de passer à une forme médiatique conçue vers l'extérieur en mettant fin à la juxtaposition des médias auparavant observée. Le portail numérique, né officiellement en janvier 2018 sous le nom de Vatican News a pour objectif d'unifier l'information vaticane en repensant les contenus, la production et la distribution de l'actualité en fonction des changements propres au numérique. Il s'agit, en définitive, pour le discours religieux de se rénover pour se conformer toujours davantage aux normes de la modernité (Douyère, 2015).

Comme le montre le schéma ${ }^{11}$ ci-dessous, l'appareil communicationnel s'appuyait jusqu'à décembre 2017 sur quatre médias principaux (Radio Vatican [RV]), l'Osservatore Romano [OR], le Centre de télévision du Vatican [CTV] et le Service internet ${ }^{12}$ ). Dès juillet 2018, l'ap-

10 Pape François, Lettre apostolique sous forme de « Motu proprio » du souverain pontife François, «Institution du secrétariat pour la communication », 2015, URL : https://w2.vatican.va/content/francesco/it/apost_letters/documents/papa-francesco_lettera-ap_20150627_segreteria-comunicazione.html

11 En bleu figurent les sites web, en vert l'application mobile.

12 C'est notamment ce service qui gère les comptes des réseaux sociaux Twitter et Instagram du pape soit respectivement @Pontifex et @franciscus, ainsi que ceux du Dicastère pour la communication@Vaticannews. 
plication mobile Vatican Audio ${ }^{13}$ a été lancée simultanément à Vatican News.

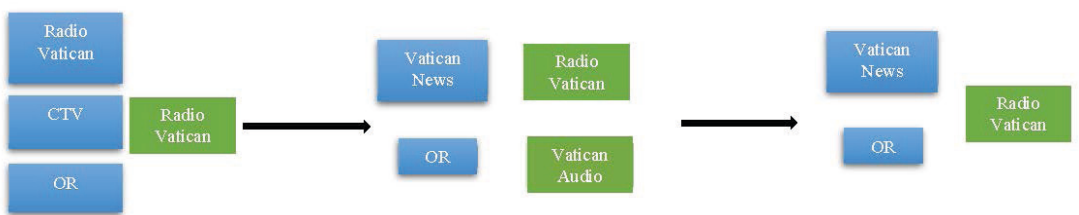

Figure 1. Schéma montrant l'évolution structurelle de l'appareil communicationnel (2016-2020) dans le cadre de la réforme des médias du Vatican (schéma réalisé par nos soins)

Avant 2018, chaque média travaillait de manière indépendante à la diffusion du contenu médiatique de l'Église catholique via son propre site web. Cette juxtaposition prend fin de façon progressive pour laisser place à une convergence médiatique au sein de laquelle les activités d'information sont coordonnées et produites ensemble.

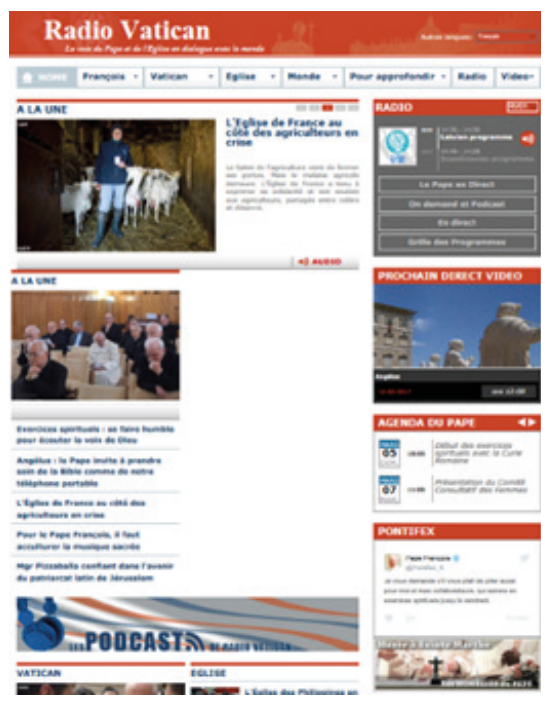

Figure 2. Capture écran de la page d'accueil du site de Radio Vatican (7 mars 2017)

13 L'application était disponible sur App Store et Google Play où l'usager choisissait la langue de l'interface, et également celle de l'écoute. Les langues étaient au nombre de 6 : italien, anglais, français, allemand, espagnol, portugais. Cette technologie est aujourd'hui intégrée à la nouvelle version de l'application mobile de Radio Vatican. 
C'est d'abord Radio Vatican, média créé le 12 février 1931 qui lance son site web (http://fr.radiovaticana.va) (cf. figure 2 ci-dessus) en 1999. Ce site couvrait, avant 2018, l'actualité vaticane, l'information catholique internationale et la vie des Églises catholiques à l'étranger.

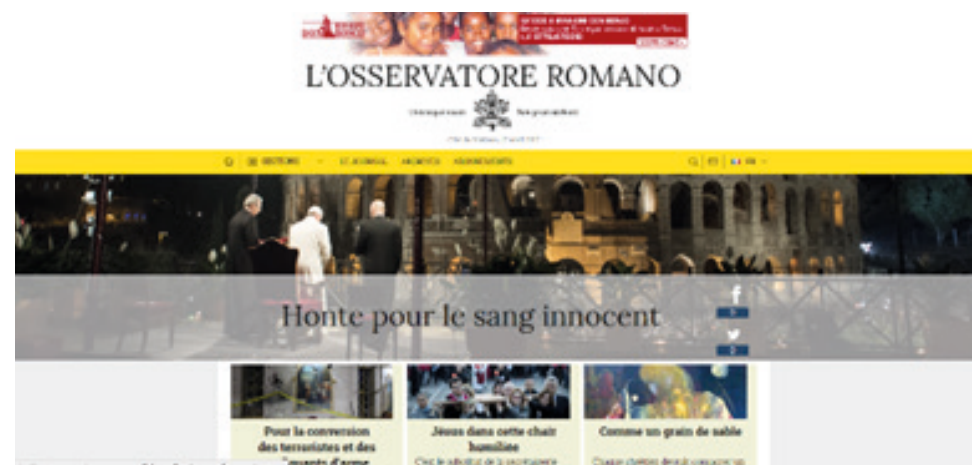

Figure 3. Capture écran de la page d'accueil du site de l'Osservatore Romano (10 juillet 2017)

L'Osservatore Romano, quotidien du Vatican, publié depuis le $1^{\text {er }}$ juillet 1861, voit son site web (https://www.osservatoreromano. va/fr.html) apparaître en ligne dans le courant de l'année 2011. C'est l'unique site du corpus ante-réforme à être encore présent en ligne tel quel aujourd'hui. Ce dernier fait l'objet d'une restructuration plus lente pour être intégré à la convergence médiatique.

Le Centre de Télévision du Vatican (CTV) a été institué en 1983 par le pape Jean-Paul II. Son site web (http://www.ctv.va) est lancé en 2013. Au cours de la convergence médiatique, l'organe d'information change de nom et est rebaptisé Vatican Media en 2017.

Le nouveau portail Vatican News devient, à partir de janvier 2018, la plateforme où convergent les contenus des anciens sites web.

Cette plateforme constitue « un cas emblématique de remédiation », " c'est-à-dire d'assimilation et d'implémentation des caractéristiques d'un medium à l'intérieur d'un autre » selon Paolo Peverini (2017). Or, les précédents sites web remédiaient déjà chaque média traditionnel du Vatican. En effet, Radio Vatican tel qu'il se présentait à l'usager entre 2016 et 2018, montrait un certain nombre de remédiations : la photographie numérique remédiait l'analogique, les podcasts remédiaient les émissions radiophoniques, les vidéos et reportages télévisés diffusés par YouTube remédiaient, via le site du CTV, les reportages réalisés par l'organe médiatique en question. Les articles sur les pages web des sites 
de l'Osservatore Romano et de Radio Vatican remédiaient quant à eux, les versions papier de la presse vaticane, etc.

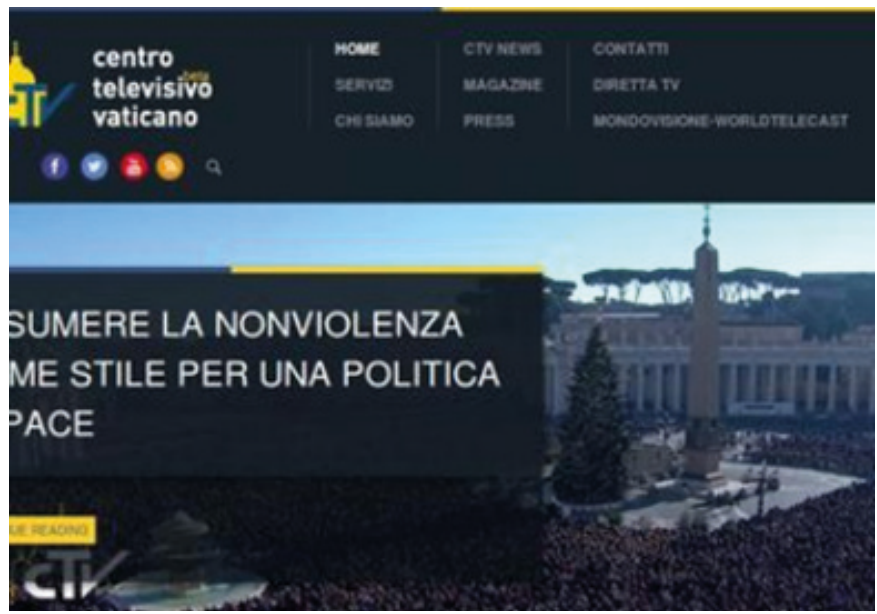

Figure 4. Capture écran de la page d'accueil du site du CTV (12 mars 2017)

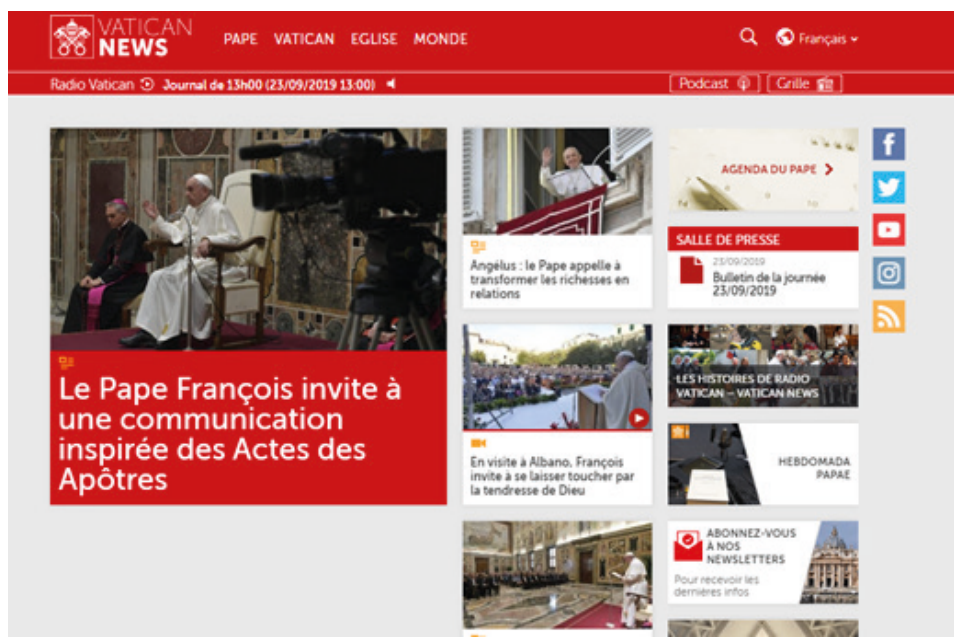

Figure 5. Capture écran de la page d'accueil de Vatican News (fr.) (23 septembre 2019)

En somme, le processus de remédiation n'apparaît pas avec la convergence médiatique. Ce qui constitue en revanche une nouveauté est le « décloisonnement des publics » entraînant avec lui « une cascade d'autres décloisonnements : entre médias, entre genres médiatiques, 
entre systèmes narratifs » (Jenkins, 2013), propres à rendre plus efficaces les leviers de visibilité du message religieux.

\section{Convergence médiatique et convergence des publics}

Le contrat de lecture est le « lien entre le document, objet fixe et circulant et les relations sociales, économiques, symboliques, qui peuvent se tisser autour de lui » (Jeanneret \& Patrin-Leclère, 2004, p. 134). Celui-ci, expliquent Yves Jeanneret et Valérie Patrin-Leclère, se charge d'une vocation publicitaire consistant à « capter des lecteurs en tant que consommateurs potentiels [...] » (Jeanneret \& PatrinLeclère, 2004, p. 138). En effet, la convergence médiatique permet au Dicastère de susciter l'intérêt d'un public qui ne soit pas seulement catholique, un public moins identifié, de masse, compétent pour diffuser et retransmettre, à l'ère de la culture participative du web 2.0, les contenus du Siège apostolique (notamment par le partage). Selon Henri Jenkins, la convergence des publics constitue une accélération et une extension de ce processus à l'âge numérique. D'ailleurs chaque site web du Vatican disposait de ses propres publics avant la convergence : le site du $C T V$ s'adressait uniquement aux italophones, celui de l'OR était élitiste clérical et celui de $R V$ davantage international. En revanche, la convergence, étape ultérieure de l'appropriation du web 2.0 par l'institution vaticane, décloisonne, comme nous allons le voir, les publics et repose sur des transformations affectant, entre autres, les codages sémiotiques et les flux de personnes.

\subsection{Quand la pratique numérique multilingue sert une traduction culturelle}

La langue est fondamentale pour l'évangélisation (Jonveaux, 2013, p. 60). Paul VI, dans l'exhortation apostolique Evangelii Nuntiandi ${ }^{14}$ émise le 8 décembre 1975, indiquait déjà que :

[...] la construction du Royaume ne peut pas ne pas emprunter des éléments de la culture et des cultures humaines. Indépendants à l'égard des cultures, Évangile et

14 Paul VI, Exhortation apostolique Evangelii Nuntiandi [en ligne] disponible sur http://w2.vatican.va/content/paul-vi/fr/apost_exhortations/documents/hf_p-vi_ exh_19751208_evangelii-nuntiandi.html 
évangélisation ne sont pas nécessairement incompatibles avec elles, mais capables de les imprégner toutes sans s'asservir à aucune. [...] Aussi faut-il faire tous les efforts en vue d'une généreuse évangélisation de la culture, plus exactement des cultures.

Comme à l'époque à laquelle Paul VI avait célébré la première messe en italien (7 mars 1965), marquant la mise en œuvre de la réforme liturgique du Concile Vatican II, l'ambition demeure encore aujourd'hui celle de transmettre les messes, prières et actualités vaticanes de façon directe, sans intermédiaire, dans le but d'établir un rapport plus immédiat, plus intime (Boni, 2002, p. 135) avec le public en imprégnant toutes les cultures. L'adaptation linguistique du discours religieux répondrait ainsi à «l'apparent et insatiable désir pour l'immédiateté » (Bolter \& Grusin, 1999, p. 9).

L'usage de la fonctionnalité numérique appelée " multilingue » n'est pas nouveau au sens qu'avant la convergence médiatique, les acteurs de la communication vaticane y avaient recours. Deux sites sur trois des médias du Vatican déclinaient leurs contenus en plusieurs langues $^{15}$. Seul celui du $C T V$ ne privilégiait cependant que les italophones.

La convergence médiatique via Vatican News permet au Dicastère d'élargir son répertoire aux langues balto-slaves, sémitiques, asliennes, altaïques et sino-tibétaines, dravidiennes, etc. notamment pour les contenus audiovisuels (disponibles auparavant seulement pour un public italophone) et de presse écrite, antérieurement limités aux seules langues italienne, allemande, anglaise, espagnole, française, polonaise, et portugaise (la plateforme Vatican News comporte une déclinaison des contenus en 38 langues ${ }^{16}$, les mêmes que celles de l'ancien site Radio Vatican auxquelles sont ajoutées le français et le portugais d'Afrique).

15 Le site de l'Osservatore Romano comptait et compte toujours sept langues : allemand, anglais, espagnol, français, italien, polonais, portugais. Celui de Radio Vatican déclinait les contenus en 36 langues : esperanto, arabe, arménien, biélorusse, bulgare, tchèque, chinois, coréen, allemand, anglais, espagnol, français, hébreu, hindi, croate, japonais, kiswahili et anglais d'Afrique, letton, lituanien, macédonien, malayalam, polonais, hongrois, portugais, portugais du Brésil, roumain, russe, albanais, slovène, slovaque, finnois, suédois, tamoul, tigrigna, ukrainien, vietnamien, amharique (parlée notamment en Éthiopie, Érythrée, Égypte, Israël, Djibouti, Yémen, Soudan).

16 Esperanto, arabe, arménien, biélorusse, bulgare, tchèque, chinois, coréen, allemand, anglais, espagnol, français, hébreu, hindi, croate, japonais, kiswahili et 


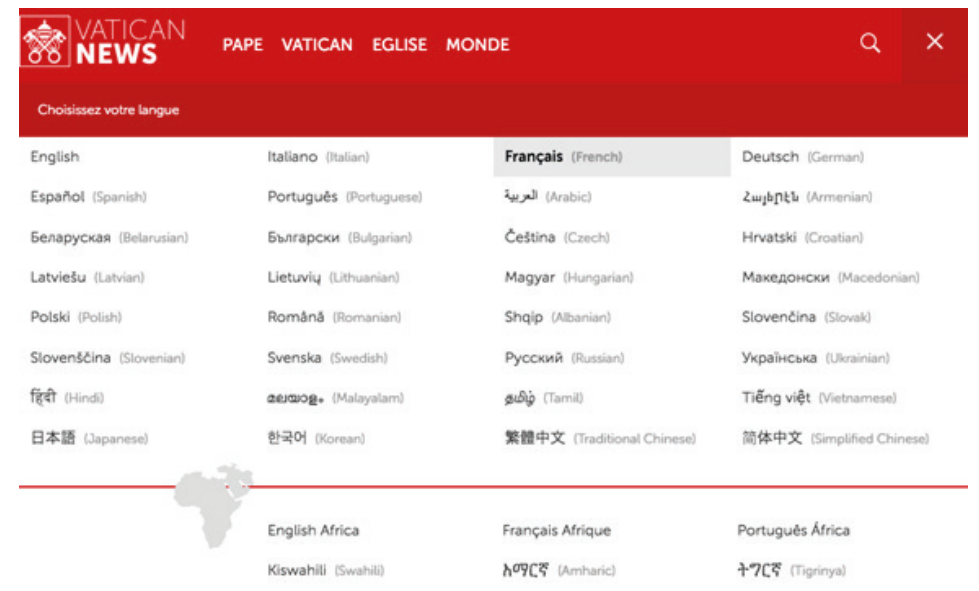

Figure 6. Pratique multilingue sur Vatican News (12 juin 2020)

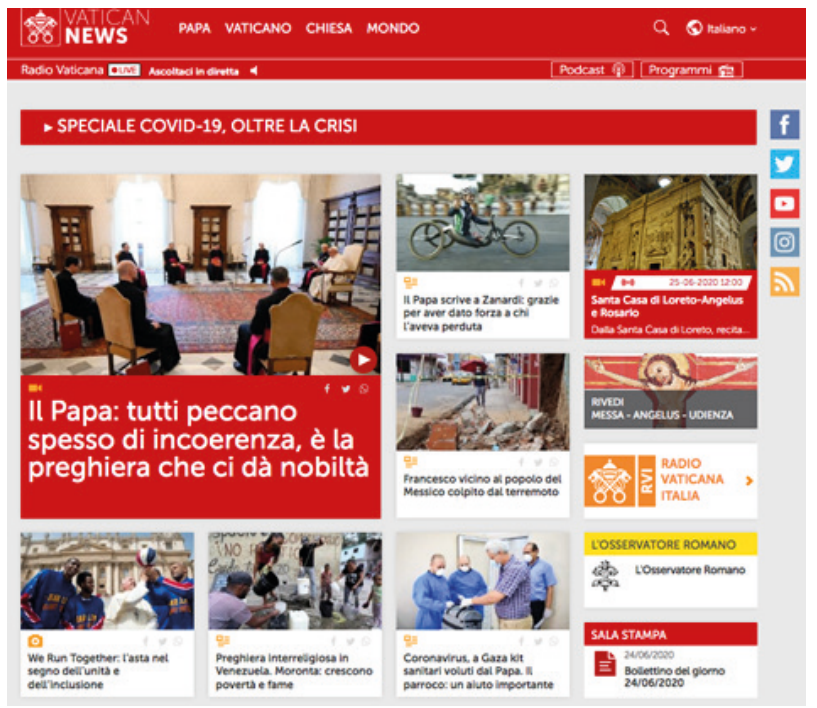

Figure 7. Une de Vatican News, version italienne (25 juin 2020)

anglais d'Afrique, letton, lituanien, macédonien, malayalam, polonais, hongrois, portugais, portugais du Brésil, roumain, russe, albanais, slovène, slovaque, finnois, suédois, tamoul, tigrigna, ukrainien, vietnamien, amharique (parlée notamment en Éthiopie, Érythrée, Égypte, Israël, Djibouti, Yémen, Soudan), français d'Afrique et portugais d'Afrique. 


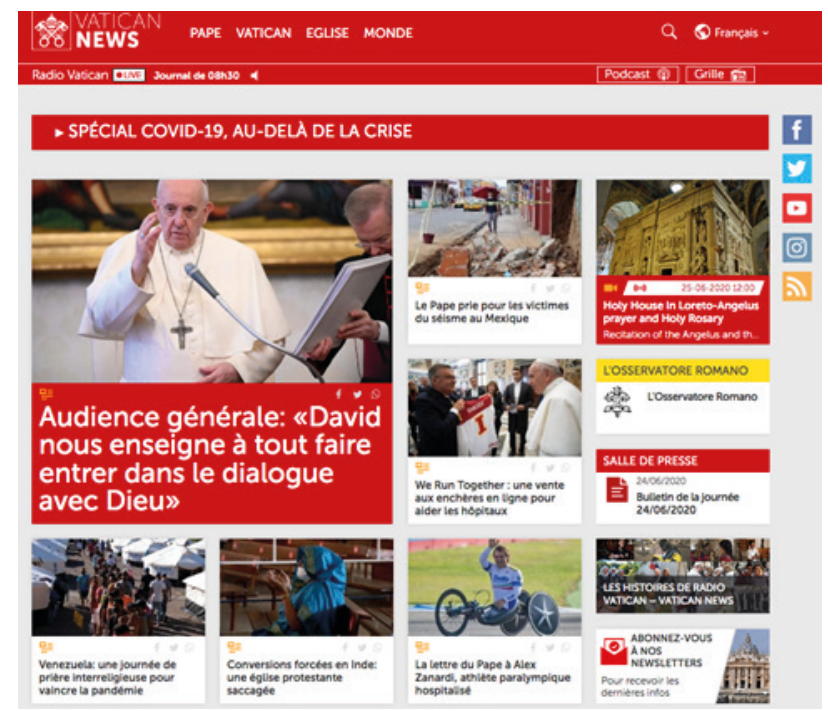

Figure 8. Une de Vatican News, version française (25 juin 2020)

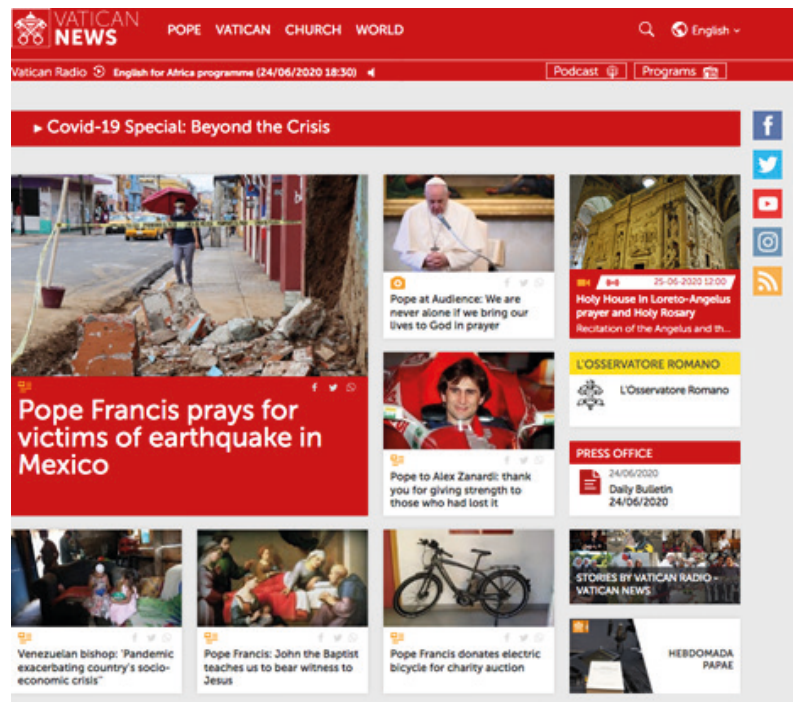

Figure 9. Une de Vatican News, version anglaise (25 juin 2020)

Toutefois, cet usage numérique du multilinguisme par les technologies du web 2.0 permet de faire autre chose qu'une « simple » traduction linguistique du discours politico-religieux. La pratique met en évidence une traduction culturelle pour mobiliser un répertoire de 
ressources qui ne sont pas seulement langagières pour satisfaire les besoins de communication et d'interaction avec un nombre étendu de personnes. Par la fonction multilingue, la page d'accueil de Vatican News adapte culturellement son contenu en fonction de la communauté linguistique et territoriale du public. Ainsi, les pages de Vatican News diffèrent les unes des autres. Les Unes italienne et française traitent, le 25 juin 2020, de l'audience générale du pape, alors que la version anglophone évoque le tremblement de terre du 23 juin au Mexique et place en second plan l'audience générale.

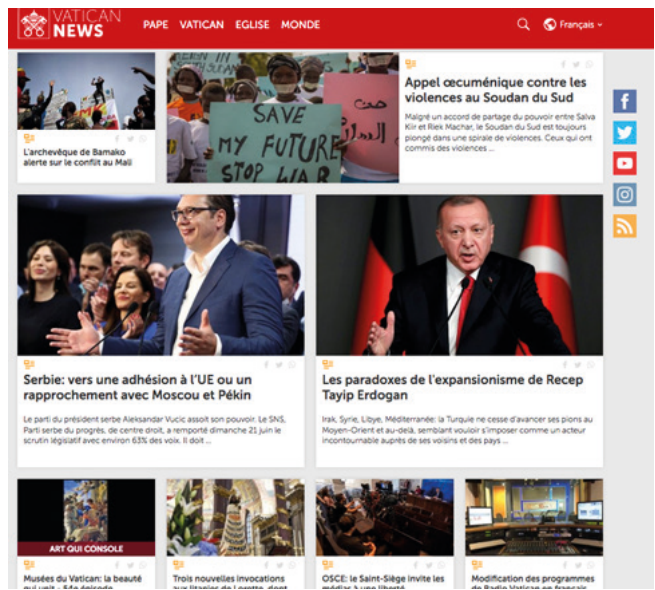

Figure 10. Zone attribuée à la politique internationale sur Vatican News en français (25 juin 2020)

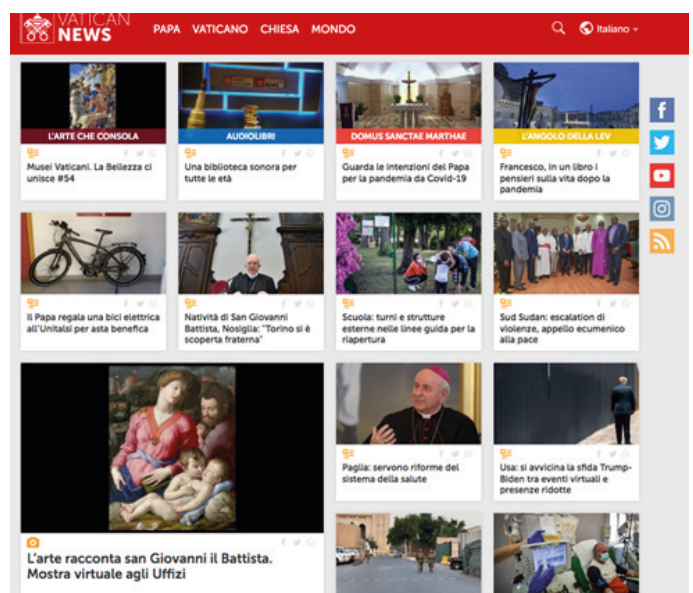

Figure 11. Zone attribuée aux productions littéraires vaticanes, aux expositions relatives aux saints à Rome sur Vatican News en italien (25 juin 2020) 


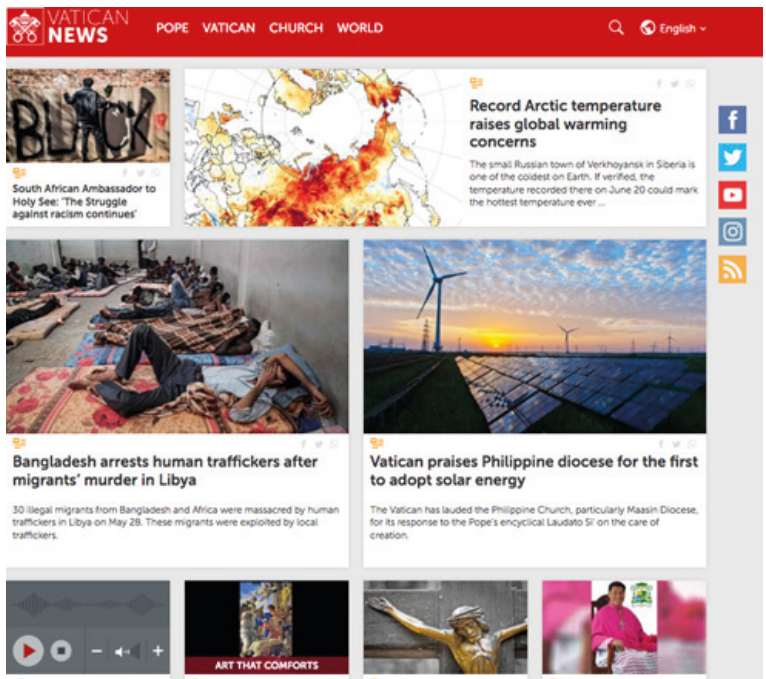

Figure 12. Zone attribuée entre autres à la thématique climatique sur Vatican News en anglais (25 juin 2020)

Une zone réservée à l'art et à la littérature sur la page italienne est supplantée par un espace dédié à la politique internationale sur la page française et une ouverture sur la thématique climatique sur la page anglaise.

Ces différences en termes de hiérarchisation de l'actualité d'une version linguistique à l'autre relèvent d'une démarche interculturelle plus que d'une ligne éditoriale spécifique à chaque langue. Étant donné que le public italophone est plus « proche » géographiquement et culturellement du Vatican, la priorité semble dans ce cas donnée à l'actualité ecclésiale tandis qu'un public anglophone représentant d'amples communautés moins identifiées géographiquement bénéficie d'une actualité plus généraliste. De même, la culture italienne, imprégnée d'une forte empreinte culturelle et artistique (l'Italie compte 55 sites inscrits au patrimoine mondial, c'est le pays le plus doté en sites du patrimoine mondial de l'humanité de l'Unesco), privilégie pour son public des contenus portant sur les arts littéraires et picturaux relatifs à l'histoire et aux saints romains.

En outre, des différences significatives apparaissent au sein d'une même langue pour signifier l'adaptation culturelle liée au territoire. Vatican News français diffère de Vatican News français d'Afrique. La page français d'Afrique regroupe les contenus correspondant à l'actualité religieuse et non religieuse des pays d'Afrique. 


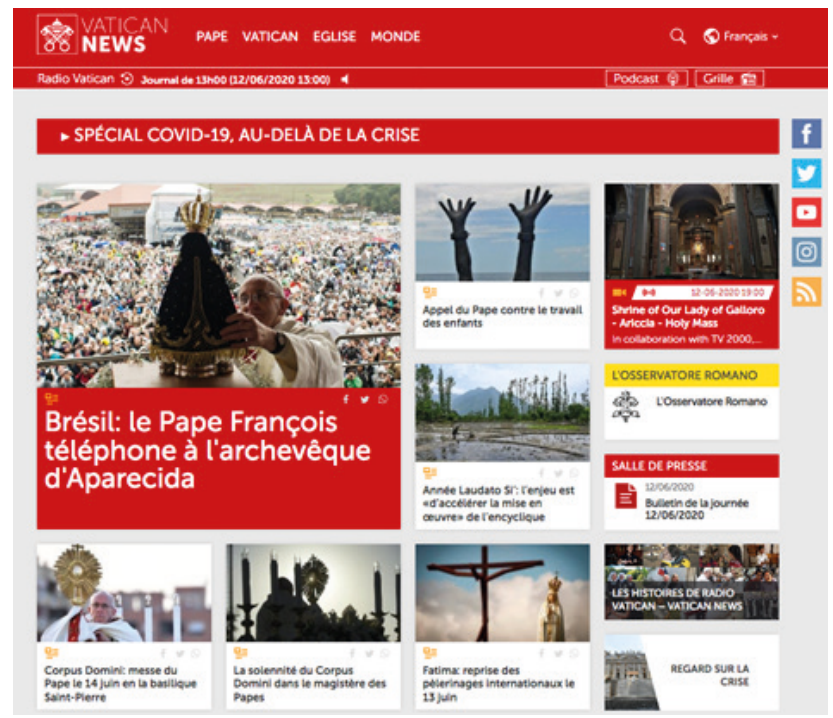

Figure 13. Page Vatican News français (12 juin 2020)

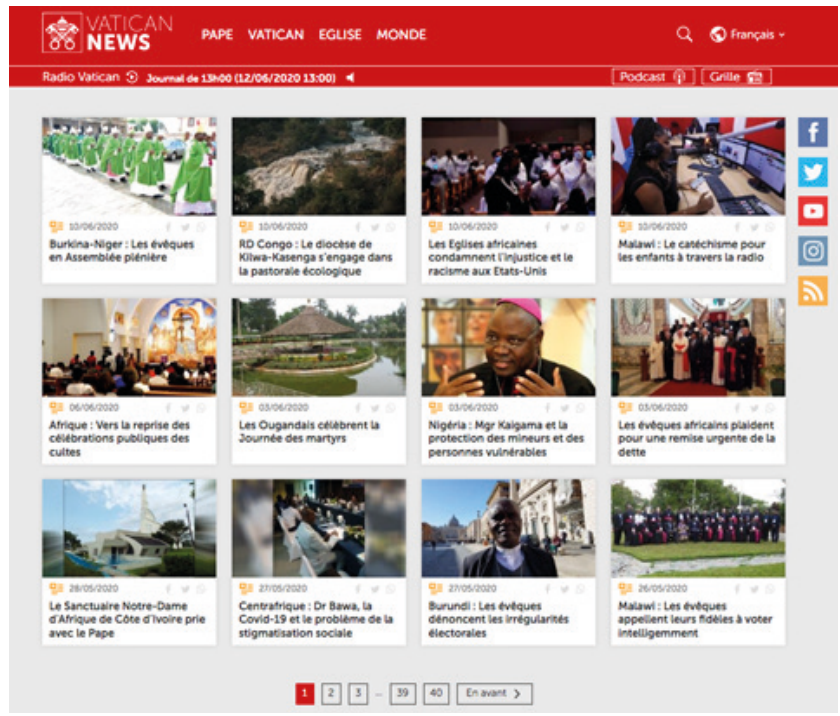

Figure 14. Page Vatican News français Afrique (12 juin 2020)

Par ailleurs la traduction linguistique des articles peut varier de l'italien (version originale) au français comme celle de la Une du 7 octobre 2018. 

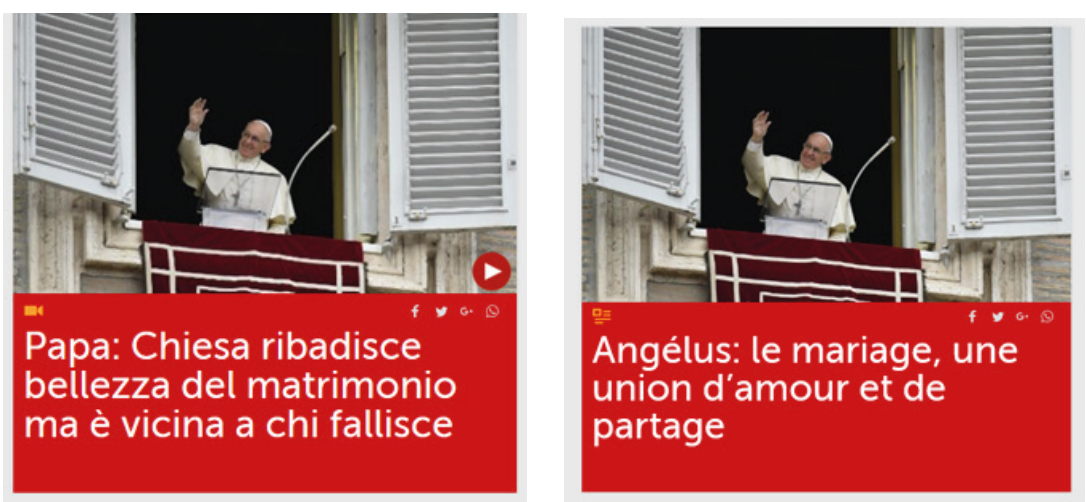

Figure 15. Version italienne et française de l'article du 7 octobre 2018

Les deux illustrations de la figure 15 montrent deux titres différents pour le même article, selon la version italienne («Pape : L'Église souligne la beauté du mariage mais est proche de ceux qui échouent ») et française ("Angélus : le mariage, une union d'amour et de partage »). Un usager italophone percevra une dimension tolérante émanant explicitement du pape envers les personnes non mariées ou divorcées, alors que le francophone l'associera davantage à la prière, au texte de l'Angelus. L'énonciateur qu'est le pape n'est pas évoqué, une distance est privilégiée, le titre se réfère au propos de la prière, au contenu. Par ailleurs, la dimension tolérante est éludée pour le discours en langue française.

Les enjeux du multilinguisme sur Vatican News ne sont pas les mêmes que sur l'application Vatican Audio. En sélectionnant sa langue, l'usager accède à tous les contenus médiatiques du jour sur l'application qui propose les discours oraux en 6 langues (italien, anglais, français, allemand, espagnol, portugais) en direct. Il s'agit dans ce cas d'une traduction linguistique.

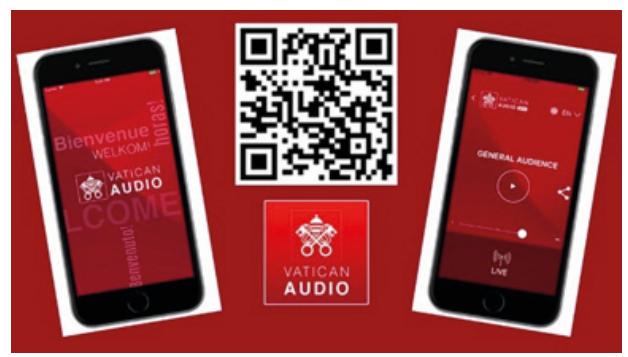

Figure 16. Application mobile Vatican Audio 
La « traductologie » vaticane s'articule donc selon deux volets : une traduction linguistique ayant notamment lieu via Vatican Audio et sur les réseaux sociaux où le discours est traduit d'une langue à une autre. Le compte Twitter du Pape est accessible en neuf langues ${ }^{17}$ et celui de @ Vaticannews en 6 langues ${ }^{18}$. De même, sur Instagram les discours sont traduits en six langues ${ }^{19}$ avant 2018 auxquelles est ajoutée la langue polonaise après cette date. En revanche, le portail Vatican News sert une traduction (inter)culturelle médiatisée selon les identités collectives des publics.

Toute culture repose sur des représentations partagées et décliner les contenus de Vatican News permet à l'institution de promouvoir, dans chaque sphère, les activités du Siège apostolique, l'actualité traitée sous l'angle catholique, ce qui, a fortiori, permet à l'institution de demeurer dans le débat public. En effet, les médias sont reliés au monde de l'opinion (Boltanski et Thévenot, 199120), où se jouent les réputations des acteurs et des institutions. En cela, l'orientation médiatique du Vatican n'est pas uniquement intra-culturelle mais multiculturelle (sa mission est universelle), et il semblerait qu'au temps de la mondialisation des échanges et de la culture du partage cette caractéristique chrétienne de multiculturalité s'accomplit de manière facilitée. La pratique multilingue permet à l'Église catholique d'étendre ses publics en dehors de l'Europe notamment, alors que son plurilinguisme via le web 2.0 est capable de faire "renaître » la langue latine ${ }^{21}$ utilisée au même titre que les langues vivantes (tweets et informations radiophoniques en latin) plus appréciée par le public que d'autres langues (Gomez Gane, 2020, p. 430).

L'intention communicationnelle est donc de faire circuler le discours en prenant en compte les cultures au sens de " totalité[s] des contenus valorisés, propres à une communauté22 $»$. En effet, dans le cas

17 Le premier compte fut ouvert en décembre 2012. À présent, il est décliné pour les langues : française, italienne, espagnole, allemande, latine, arabe, polonaise et portugaise.

18 Anglais, espagnol, italien, portugais, français, et allemand.

19 Anglais, espagnol, italien, portugais, français, et allemand.

20 Catherine Roth \& Carsten Wilhelm (2016) citent Luc Boltanski et Laurent Thévenot, De la justification, Paris, Gallimard, 1991.

21 La rubrique « Prie avec le pape » propose des prières en latin et @Pontifex_in publie des tweets journaliers en latin.

22 Définition sémiotique du terme «culture » que donne Algirdas J. Greimas (1976, pp. 45-46). 
de la traductologie, l'Église catholique semble concevoir les cultures en tant qu' " ensembles des phénomènes matériels et idéologiques qui caractérisent un groupe ethnique ou une nation, une civilisation, par opposition à un autre groupe ou à une autre nation ${ }^{23} \gg$.

Ainsi, nous pouvons conclure que la multiculturalité est une valeur ajoutée à la pratique multilingue de la part de l'institution vaticane. Elle incarne une valeur de médiation, au sens que le multiculturalisme constitue une dimension nouvelle ${ }^{24}$ pour la médiation technologique du discours politico-religieux de l'Église catholique.

\subsection{Des publics culturellement différents : du vaticaniste au moins averti en termes de culture religieuse}

Avec l'apparition de Vatican News, de nouvelles pratiques rhétoriques émergent. Selon Paolo Peverini (2017, p. 115), le portail préfigure des « usages modèles » conçus en fonction de profils d'usagers.

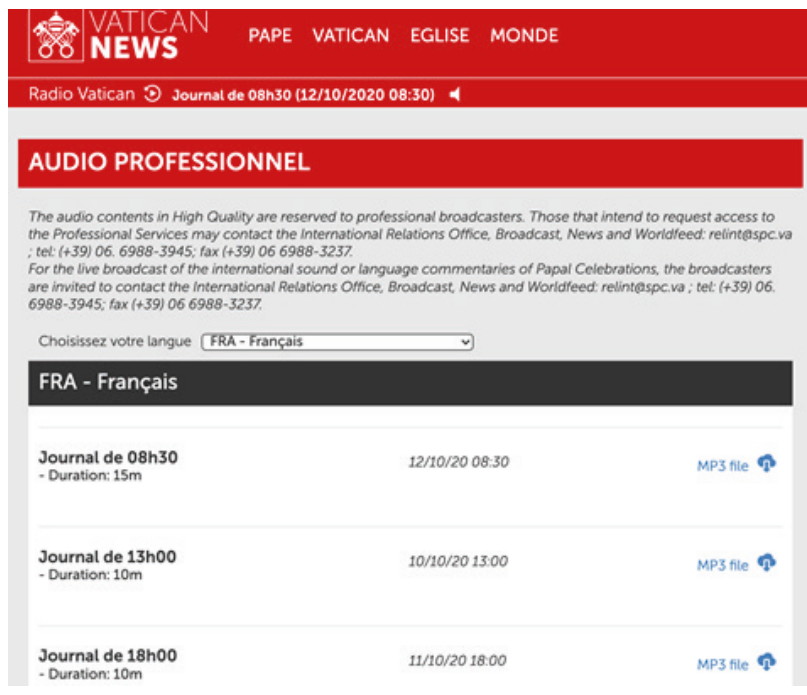

Figure 17. Section « Audio professionnel » sur Vatican News

23 Définition de " culture » sur le site de Larousse https://www.larousse.fr/dictionnaires/francais/culture/21072.

24 Yves Jeanneret (2014) définit la médiation en tant qu' « activité productive et créative qui consiste à intervenir sur le cours de la communication en lui apportant une dimension nouvelle». 
Plusieurs types se dessinent allant du vaticaniste à un public plus profane en termes de culture religieuse. La rubrique « audio professionnel » vise, comme indiqué sur Vatican News, à informer des spécialistes, professionnels des médias afin que ces derniers puissent relayer les informations du Siège apostolique. La rubrique du bulletin de presse s'adresse également à un public averti, vaticaniste pouvant s'informer sur les conférences de presse, les nominations et démissions au sein de l'Église catholique, entre autres.

La section des prières se destine à un public pratiquant pouvant s'appuyer sur les textes et enregistrements audio. Ce dernier peut aussi visionner en direct l'Angelus, la messe.

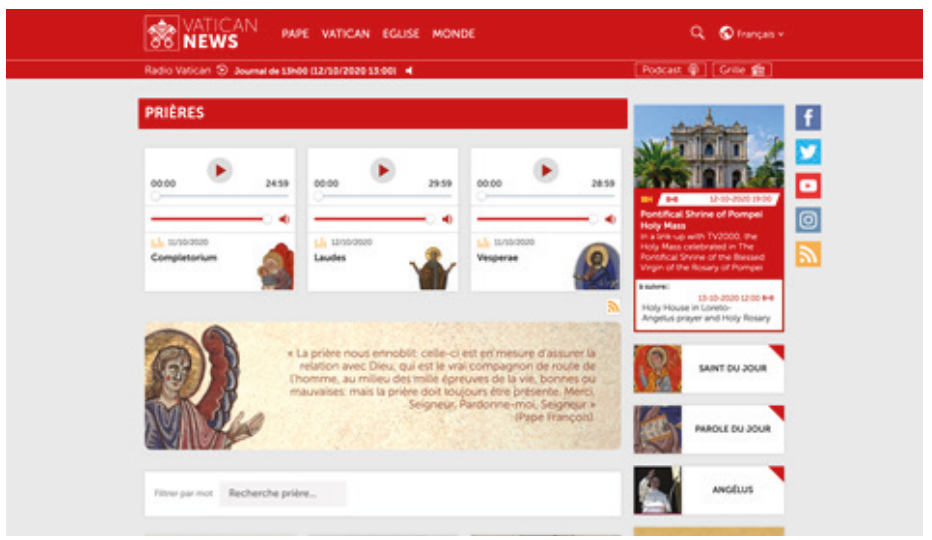

Figure 18. Section «prières » sur Vatican News

Le public fidèle demeure une cible importante de Vatican News, notamment à travers la mise au service de contenus spécifiques - en langue latine - pour prier, s'informer.

Le portail d'information montre par ailleurs une ouverture " didactique », une intention de communiquer en vulgarisant, d'initier un public éloigné, voire étranger (peut-être novice, profane) à la culture religieuse - que ce dernier soit chrétien ou non - en tous cas semblant être dépourvu d'une encyclopédie ${ }^{25}$ chrétienne. L'énoncé « qu'est-ce que l'Angelus ? » tend à apprendre à qui n'est pas initié, une notion catholique classique.

25 Selon Umberto Eco (Lector in fabula), l'encyclopédie se réfère à l'ensemble de savoirs, connaissances et compétences de l'individu. 
Regina Coeli

Regina coeli, wetare, alleluis.

Quia quem mervisti portare, alleluis.

Resurrexit, sicut doct, alleluia.

Orapro nobis Deum, alleluis.

$R$. Quas surrext Dominus vere, alleluia.

Oremus:

Deus, qui per resurrectionem filli tui Domini nostri lesu

Christi mundum laetificare dignatus es, praesta.

quaesumus, ut per eius Genetricem Virginem Mariam

perpetuae caplamus gaudia vitae.

Per eundem Christum Dominum nostrum. Amen.
V. Gaude et isetare, Virgo Maria. alleluia

\section{Regina Coeli}

Reine du ciel rejouissez-vous, alletuis

car Celui que vous avez merite de porter dans votre sein alleluia

est ressuscite comme llra dit alletus

Priez Dieu pour nous, allelluis.

Soyez dans la joic et raliegresse, Vierge Marie, alletula. Parce que le Seigneur est vraiment ressuscite, alleluia Prions

Deu, qui par is Resurrection de Votre Fils, notre

Seigneur Jesus-Christ, avez bien voulu rejouir le monde.

Faites-nous parvenir, par la pribere de la Vierge Marie, sa

mere, aux joies de la vie eternelle. Par le Christ notre

Seigneur.

Amen.

\section{$\frac{n}{3}$}

Figure 19. Prière bilingue latin-français Regina Coeli (25 juin 2020)

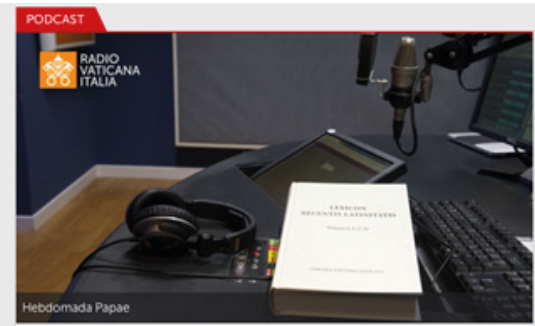

\section{Hebdomada Papae}

Notitiae vaticanae latine redditae. La settimana del Papa, notiziario vaticano n lingua latina
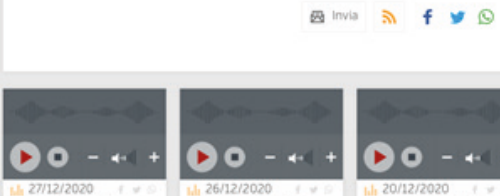

Rasiogornale Latino

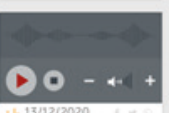

Radiogicanale Latin

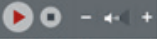

Radiogionale Latino

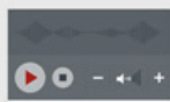

(4) $08 / 12 / 2020$ Radiogiomnale Latino
08122020
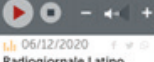
Radiogiornale Latino
06.12 .2020

12 - 1 9

Figure 20. Hebdomada Papae : le journal d'information du Vatican en latin (3 janvier 2021) 


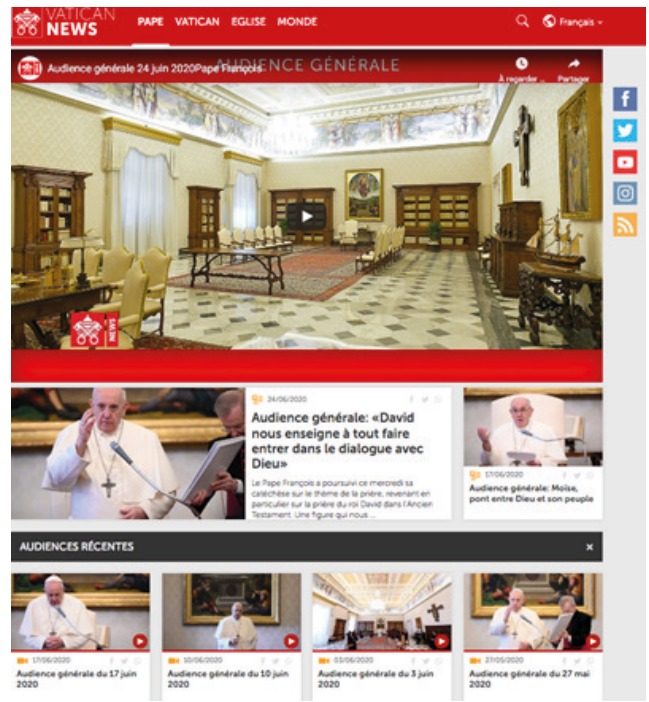

Figure 21. Les discours de l'audience générale constituent une rubrique importante (26 juin 2020)

Figure 22. Menu de l'Angelus où apparaît une note explicative sur Vatican News

LAngelus est une prière en mémoire du Mystère de IIncarnation du Christ, récitée trois fois par jour : a six heures le matin, a midi et le soir vers dixhuit heures, au moment où sonne la cloche de l'Angelus. Son nom Angelus derive du premier verset de la priere en latin: Angelus Domini nuntiavit Mariae Elle consiste en une lecture brève de trois textes simples sur l'Incarnation du Christ, et la récitation de trois s Je vous salue Maries. Cette prière est recitee par le Pape tous les dimanches et les jours de fete a midi place Saint Pierre. Avant de réciter la priere. le Pape prononce un bref discours se référant aux Lectures du jour. Après la prière, il salue les pèlerins.

Entre Paques et Pentecôte, l'Angellus céde sa place au Regina Coeli, une priere qui fait mémoire de la résurrection de Jesus Christ et a la fine de laquelle le Gloria est récité trois fois.

Figure 23. Explication de ce qu'est l'Angelus sur Vatican News 
L'ouverture axiologique impliquée par des publications de Vatican News via d'autres dispositifs confirme cette intention de parler à des individus éloignés de la culture catholique et plus proche d'une culture populaire. Les posts du Dicastère sur Instagram en témoignent. La communication ad extra pour l'institution doit en effet permettre de rejoindre «le commun des mortels » (Fumagalli, 2015, p. 59), autrement dit, des groupes d'individus qui ne sont pas nécessairement proches de la culture catholique.

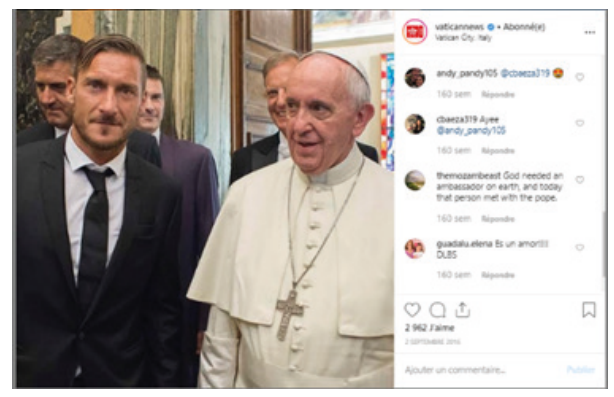

Figure 24. Rencontre du pape et de Francesco Totti au Vatican (2 septembre 2016) Source : https://www.instagram.com/vaticannews/

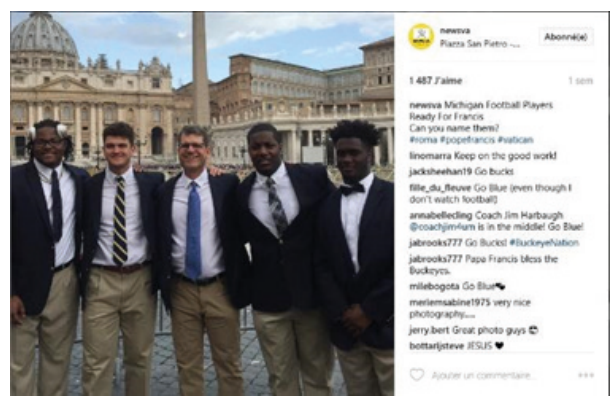

Figure 25. L'équipe des joueurs de football du Michigan au Vatican (28 avil 2017) Source : ancien compte Instagram@Newsva 


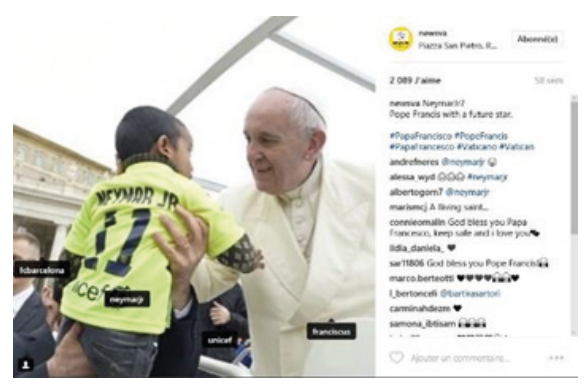

Figure 26. Post humoristique associant un enfant au joueur de football Neymar "Pope Francis with a future star" (5 mai 2017) Source : ancien compteInstagram@Newsva

\section{(aPONTIFEX_FR}

Seul celui qui regarde avec le cœeur voit bien, car il sait «voir audedansa: la personne au-dela de ses erreurs, le frère au-dela de ses fragilités, l'espérance dans les difficultés. Dieu en tous.

Tout afficher ?
(0) aFRANCISCUS

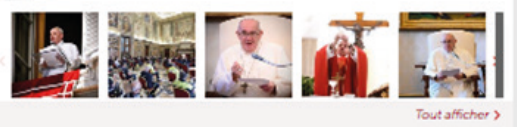

Figure 27. Encarts des réseaux sociaux faisant apparaître les contenus et liens vers les comptes Twitter et Instagram du pape (23 juin 2020)

Un encart sur le site Vatican News où apparaissent les dernières publications sur les réseaux sociaux fait le lien entre l'usager de la plateforme des médias convergés et les autres technologies.

De plus, Vatican News présente une forte dimension multimédia : texte écrit, photographie et vidéo sont pleinement intégrés et l'information vaticane se transmet désormais selon une multitude de langages et de manifestations (verbales, visuelles, audiovisuelles). La multimédialité explicite sur le portail d'information favorise une multimodalité rendant l'expérience de l'usager plus complète. Cette dernière prend de la valeur par les pictogrammes orange indiquant à l'usager le canal de réception informationnelle :

\begin{tabular}{|c|c|c|c|}
\hline 먀 $11 / 02 / 2019 \quad$ f $\vee Q 9$ & () $11 / 02 / 2019$ is & h. $22 / 06 / 2019$ & $=121 / 04 / 2019$ \\
\hline $\begin{array}{l}\text { Une délégation de } \\
\text { l'opposition vénézuéllenne } \\
\text { reçue au Vatican }\end{array}$ & $\begin{array}{l}\text { Il y a } 91 \text { ans, la signature des } \\
\text { Accords du Latran }\end{array}$ & $\begin{array}{l}\text { Journal en latin de Radio } \\
\text { Vatican 22.06.2019 }\end{array}$ & $\begin{array}{l}\text { Paques 1949: pour la première } \\
\text { fois, le Pape parle en français } \\
\text { à ta télévision }\end{array}$ \\
\hline
\end{tabular}

Figure 28. Exemples de valorisation des canaux utilisés sur Vatican News 
Ce caractère renforcé du multimédia permet de toucher plusieurs types de publics selon une logique de profils allant du plus initié au plus profane comme l'évoque Dario Edoardo Viganò :

Les textes peuvent constituer un approfondissement pour une personne qui est plus préparée, plus avertie en matière religieuse alors qu'un public jeune peut préférer la vidéo à l'article, l'oralité étant en effet paratactique et suscitant plus d'empathie par rapport à l'écriture. La multimédialité sert aussi cela : toucher plusieurs types de personnes ${ }^{26}$.

En outre, la déclinaison médiatique est valorisée par la manipulation que peut effectuer l'usager en réalisant une recherche de contenus :

\begin{tabular}{lr} 
TYPE & $\mathbf{x}$ \\
$\square$ ARTICLE & 2897 \\
$\square$ VIDÉO & 268 \\
$\square$ PHOTO & 129 \\
$\square$ AUDIO & 2 \\
\hline
\end{tabular}

Figure 29. Déclinaison médiatique pour l'usager sur Vatican News

D'ailleurs Henri Jenkins évoque lui aussi le rapport entre public jeune et médium de l'image :

26 Commentaire de Dario Edoardo Viganò, assesseur du Dicastère recueilli le 17 décembre 2018 lors d'un entretien semi-directif dans les locaux du Dicastère pour la communication (Via della Conciliazione à Rome). Nous avions défini en amont les questions ouvertes à poser au regard des résultats après une étude sémiotique des pratiques numériques institutionnelles (privilège de certains dispositifs, usages de fonctionnalités plutôt que d'autres, etc.). Les réponses, libres, ont été enregistrées puis traduites et transcrites en français. Ces entretiens ont permis d'apporter des informations complémentaires de background (contexte et intentionnalité des pratiques) à l'étude, pour laquelle il s'agit de considérer l'importance des attitudes, des perceptions et des comportements de l'institution. Une telle méthode d'entretiens semi-directifs peut présenter certaines limites du fait de l'orientation (cadrage) du débat et de ses éventuels manquements. Les propos d'un acteur institutionnel du Vatican nécessitent par ailleurs une vision critique des réponses, notamment au regard des notions d'intention, de stratégies communicationnelles, souvent perçues péjorativement et défavorisées au profit de l'évocation d'une motivation divine pour la communication. 
Pour un nombre croissant de jeunes, l'image (ou plus précisément, la combinaison de mots et d'images) constitue un ensemble de ressources rhétoriques aussi important que des textes.

La plateforme Vatican News tout comme son compte sur Instagram ambitionne de toucher ce public, notamment en figurant le pape aux côtés de jeunes faisant des selfies avec lui.

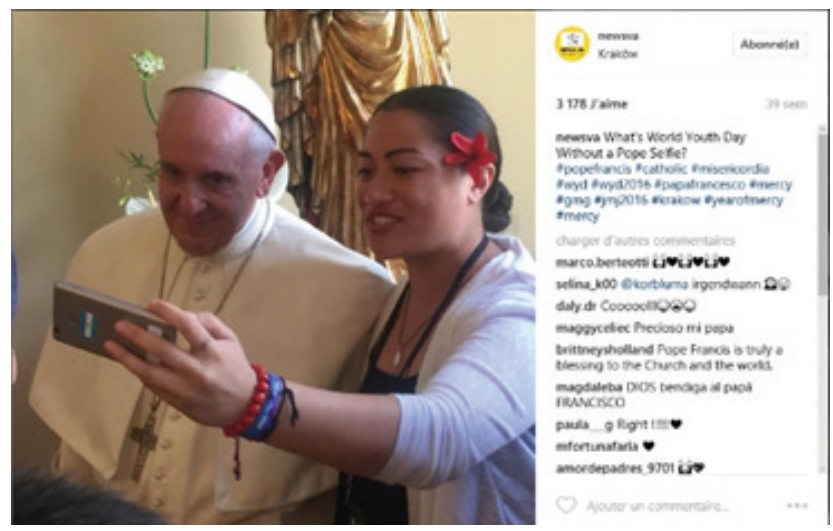

Figure 30. Le pape et une jeune fille faisant un selfie durant la journée mondiale de la jeunesse en 2016. Source : ancien compte Instagram@Newsva

Cette dernière partie nous permet de constater que la convergence des publics se manifeste à travers un critère autre que linguistique. En effet, la pratique institutionnelle tend à considérer les publics sous une seconde acception du terme " culture », à savoir celle qui renvoie plus particulièrement aux groupes sociaux.

\section{Conclusion}

Depuis la fin des années 1990, l'institution vaticane ne cesse d'actualiser ses pratiques numériques pour faire circuler amplement son discours à travers sa cybercampagne. Dès la fin de l'année 2017, l'assesseur du Dicastère expliquait l'intention communicationnelle de :

Privilégier une communication multimédia, multilinguistique et multiculturelle pour répondre aux divisions linguistiques des publics par des traductions de la perception 
culturelle et non pas par de simples «traductions » des discours.

En questionnant les formes médiatiques et notamment le phénomène de convergence, nous avons identifié une traductologie fondée simultanément sur une valeur multilingue et sur une valeur multiculturelle (tant celle attachée aux groupes ethniques et nationaux que celle reliée à la culture au sens de groupe social).

À la lumière du travail d'Henri Jenkins, cette étude a permis de déterminer en quoi la convergence médiatique engendre celle des publics, actualisant ainsi résolument à l'ère numérique la mission ecclésiale ambitionnant de "parler à tous les Hommes » au-delà de leur langue et de leur culture, privilégiant une immédiateté pour la diffusion du message religieux.

L'ambition est celle de s'adresser à un public universel où l'universalité est entendue à la fois comme une dimension internationale et à la fois en ce qu'elle concerne, implique l'inclusion de tous les hommes, qu'ils soient proches ou non de la culture religieuse, qu'ils soient vaticanistes, professionnels, catholiques pratiquants ou même profanes. Les pratiques médiatiques désormais numériques, semblent donc, via les fonctionnalités du web 2.0, réactualiser l'universalité au sens étymologique de $\kappa \alpha \theta 0 \lambda \imath \kappa o ́ s$, et par là même favoriser une quête de " popularité » dans les arènes numériques. L'universalité du langage à travers le web implique simultanément plusieurs codes, ceux de la culture catholique et ceux d'une culture plus populaire comme c'est le cas sur Instagram.

Ainsi, alors que la déclinaison cross media produisait la dispersion et le cloisonnement des publics selon une logique de juxtaposition médiatique la plateforme Vatican News gagne en revanche en valeur médiatique pour le discours institutionnel par rapport à l'ancienne application mobile Vatican Audio et aux sites web de Radio Vatican et du $C T V$. Cette restructuration médiatique se fonde sur la double logique de la remédiation en ce qu'au fil du temps, le désir d'immédiateté anime la réorganisation de l'écosystème des médias du Vatican. Ce dernier se voit davantage hypermédié et les déclinaisons linguistique et culturelle engendrent inévitablement une visibilité des productions médiatiques vaticanes qui pour certaines communautés linguistiques et certains groupes sociaux ne l'étaient pas auparavant.

Enfin, le nouvel écosystème Vatican News répond aussi à la nécessité de changement de l'identité institutionnelle qui doit demeurer en 
accord avec les mutations communicationnelles contemporaines afin de transmettre son message dans les sociétés modernes.

\section{Références}

Bolter, J.D. \& Grusin, R. (1999). Remediation. Understanding New Media. Cambridge, MA : The MIT Press.

Boni, F. (2002). Il corpo mediale del leader. Rituali del potere della sacralità del corpo nell'epoca della comunicazione globale. Coll. Nautilus. Rome : Meltemi editore.

Bougnoux, D. (1995). La Communication contre l'information. Paris : Hachette.

Campbell, H.A. (2017). Surveying Theoretical Approaches within Digital Religion Studies. New Media \& Society, 19(1), 15-24. Doi : https://doi. org/10.1177/1461444816649912

Douyère, D. (2015). Les mutations communicationnelles du christianisme catholique $\mathrm{au} \mathrm{XX}^{\mathrm{e}}$ siècle. Hermès 71. Le XX $\mathrm{X}^{e}$ siècle saisi par la communication. Vol. 2 « Rupture et filiations », 225-235.

Eco, U. (1985). Lector in fabula. Le rôle du lecteur [1979]. Paris : Grasset \& Fasquelle.

Fontanille, J. (2006). Pratiques sémiotiques : immanence et pertinence, efficience et optimisation. Nouveaux actes sémiotiques, 104-106, 13-75.

Fumagalli, A. (2015). La comunicazione di una «chiesa in uscita». Riflessioni e proposte. Milan : Vita e pensiero.

Gomez Gane, Y. (2020). @Pontifex: La Santa Sede tra latino, italiano e le altre lingue. Dans V. Sanzotta (Ed.), Une lingua morta per letterature vive: il dibattito sul latino come lingua letteraria in età moderna contemporanea. Atti del Convegno internazionale di studi Roma 10-12 dicembre 2015 (pp. 409-431). Coll. Supplementa Humanistica Lovaniensia, XLIV. Louvain: Leuven University Press.

Greimas, A. J. (1976). Sémiotique et sciences sociales. Paris : Seuil.

Jeanneret, Y. (2014). Critique de la trivialité. Les médiations de la communication, enjeu de pouvoir. Paris : Non Standard.

Jeanneret, Y. \& Patrin-Leclère, V. (2004). La métaphore du contrat. Hermès, 38. Les sciences de l'information et de la communication savoirs et pouvoirs, 133-140.

Jenkins, H. (2013). La Culture de la convergence. Des médias au transmédia. Coll. Médiacultures. Paris : Ina éditions/A. Colin.

Jonveaux, I. (2013). Dieu en ligne. Expériences et pratiques religieuses sur internet. Paris : Bayard.

Mitropoulou, E. (2015). Valeur et sémiotique de la communication médiatée : pour une approche inférentielle et transversale. Dans A. Biglari (Ed.), Valeurs. Aux fondements de la sémiotique (pp. 579-607). Paris : L’Harmattan.

Pape François (2015). Lettre apostolique sous forme de « Motu proprio » du souverain pontife François, «Institution du secrétariat pour la communication ». Disponible à https://w2.vatican.va/content/francesco/it/apost_letters/documents/papa-francesco_lettera-ap_20150627_segreteria-comunicazione.html

Pape Paul VI (1975). Exhortation apostolique sur l'évangélisation dans le monde moderne « Evangelii Nuntiandi ». Disponible à : w2.vatican.va/content/paul-vi/fr/ apost_exhortations/documents/hf_p-vi_exh_19751208_evangelii-nuntiandi.html 
Papi, C. (2018). Médiations et médiatisation, entretien avec Daniel Peraya. Médiations et médiatisations, 1(1), 102-111. Disponible à : https://revue-mediations.teluq.ca/ index.php/Distances/article/view/61

Paveau, M.-A. (2017). L'Analyse du discours numérique. Dictionnaire des formes et des pratiques. Paris : Éditions Hermann.

Peverini, P. (2017). Francesco e i media vaticani. Un racconto in divenire. Dans A.-M. Lorusso \& P. Peverini (Eds.), Il racconto di Francesco. La comunicazione del papa nell'era della connessione globale (pp. 113-134). Rome : Luiss University Press.

Roth, C. \& Wilhelm, C. (2016). Médias culturels et Interculturalité : regards croisés France-Allemagne. Revue française des sciences de l'information et de la communication, 9. Doi: https://doi.org/10.4000/rfsic.2451

Souchier, E., Jeanneret, Y. \& Le Marec, J. (2003). Lire, écrire, récrire : objet, signes et pratiques des médias informatisés. Paris : Bibliothèque Centre Pompidou. 


\section{(c) (1) $(9)$}

BY NC ND Publié sous la licence Creative Common

«Attibution - pas d'utilisation Commerciale - Pas de Modification 4.0. International» (CC BY-NC-ND) 